\title{
Caractérisation de la résistance au froid de deux espèces sclérophylles méditerranéennes par la fluorescence de la chlorophylle
}

\author{
M Méthy*, L Trabaud \\ CNRS, Centre d'écologie fonctionnelle et évolutive, BP 5051, 34033 Montpellier Cedex. France
}

(Reçu le 16 février 1989 ; accepté le 16 mai 1989)

\begin{abstract}
Résumé - La capacité de résistance au froid du système photosynthétique de Quercus ilex et Quercus coccifera, 2 espèces sclérophylles de la région méditerranéenne, a été étudiée. Les cinétiques d'induction de la fluorescence de la chlorophylle ont été analysées sur des feuilles âgees de 1 an et sur des jeunes feuilles âgees de 1 mois. Pour les 2 espèces et pour les 2 stades de développement considérés, la réduction de la réponse observée est très rapide. Bien qu'apparaissant comme le plus résistant, le système photosynthétique de $Q$ ilex est détruit, sans possibilité de récupération, après 30 min d'exposition à $-20^{\circ} \mathrm{C}$. L'impact des températures hivernales sur les aires de répartition de $Q$ ilex et $Q$ coccifera est évoqué.
\end{abstract}

fluorescence de la chlorophylle / gel / Quercus ilex / Quercus coccifera / sclérophylle

Summary - Frost resistance characterisation of two mediterranean sclerophyllous species by chlorophyll fluorescence. Frost resistance of the photosynthetic system of 2 sclerophyllous species of the mediterranean region, Quercus ilex and Quercus coccifera, has been analysed. Measurements were made on 1 year and 1 month old leaves by the chlorophyll fluorescence induction method. Photosynthetic activity is inhibited very quickly for both the 2 species at the development stages studied. The photosynthetic system of $Q$ ilex seems to be the most resistant but is destroyed after half an hour of exposure to cold at $-20^{\circ} \mathrm{C}$, without any recovery after treatment. The effect of winter temperatures on the distribution areas of $Q$ ilex and $Q$ coccifera is discussed.

chlorophyll fluorescence / freezing / Quercus ilex / Quercus coccifera / sclerophyll

\section{INTRODUCTION}

Quercus ilex et Quercus coccifera sont 2 espèces de chêne vivant dans l'aire

Correspondance et tirés à part du climat méditerranéen où elles constituent des peuplements forestiers importants. Ce sont des végétaux sclérophylles et sempervirents. Ces espèces ligneuses dominantes des garri- 
gues prolongent, avec un feuillage persistant, leur photosynthèse toute l'année, équilibrant ainsi un bilan énergétique amoindri par une respiration estivale importante (Eckardt et al, 1975). En conditions naturelles, de nouvelles pousses et des jeunes feuilles apparaissent, le plus souvent, vers les mois d'avril et mai.

Au cours des mois d'hiver, des froids parfois intenses (avec, par exemple, des températures de $-25^{\circ} \mathrm{C}$ en 1956 et 1965 enregistrées dans la région de Montpellier) peuvent provoquer des destructions sur les végétaux: gel des jeunes pousses, parfois même mort d'individus. La présence de feuilles persistantes reflète cependant une certaine résistance au froid du système photosynthétique de ces 2 espèces ; mais, au printemps, quand la photosynthèse apparente présente son maximum, les jeunes pousses se trouvent elles aussi exposées à un éventuel brusque abaissement de la température.

En outre, en toute saison, des coupes d'exploitation ou des incendies - 2 types de traumatisme très fréquents dans la région méditerranéenne (Trabaud, 1981) - peuvent faire disparaître toute la partie épigée de ces espèces qui, alors, vont produire des rejets portant des jeunes feuilles. Quelle sera l'action du froid sur le système photosynthétique des feuilles (vieilles ou jeunes)? Est-ce que la résistance de l'appareil sera la même pour les 2 stades de développement? Permettra-t-il aux rejets de survivre, ou seront-ils tués, et la partie souterraine de la plante obligée de produire de nouvelles pousses portant un nouvel appareil chlorophyllien? Les réponses à ces questions peuvent apporter quelques informations sur les capacités de survie de ces espèces forestières lorsqu'elles poussent en limite de leur aire d'extension normale.
En dehors des résultats relatifs aux espèces sclérophylles méditerranéennes, classées du point de vue de leur résistance au froid par Sakai et Larcher (1987), peu de travaux semblent avoir été réalisés sur ces questions. Il est à noter, toutefois, une étude approfondie de Larcher (1970) à propos de quelques-unes de ces especes, mais le rôle de la durée d'application du froid n'est pas abordé.

Le présent travail est donc destiné à apprécier l'impact de ce stress sur le système photosynthétique lui-même de feuilles âgées de 1 an (vieilles feuilles) ou de 1 mois (jeunes feuilles) de $Q$ ilex et $Q$ coccifera, avant même l'apparition de symptômes décelables visuellement.

\section{MATÉRIELS ET MÉTHODES}

Les méthodes basées sur l'analyse de l'induction de la fluorescence de la chlorophylle sont connues pour bien se prêter à la détection précoce des stress chez les végétaux (Lichtenthaler et Rinderle, 1988). Pour un végétal photosynthétiquement actif placé prealablement à l'obscurité durant quelques dizaines de minutes, la cinétique d'induction de la fluorescence, à l'origine décrite par Kautsky et Hirsch (1931), comporte l'aspect et les différentes phases indiquées dans la figure 1 , en relation avec l'échelle de temps mise en œuvre.

Durant les premières secondes, on peut observer une phase ascendante (OIDP) avec passage rapide d'un niveau initial $(O)$ à un maximum $(P)$. La fluorescence décroît ensuite jusqu'à un état quasi stationnnaire (S), puis augmente à nouveau pour atteindre un deuxième maximum (M), d'où elle decroît ensuite lentement vers un niveau final ( $T$ ). Ces phases sont en relation avec les changements intervenant dans les fonctions photosynthétiques (Lavorel et Etienne, 1977).

C'est ainsi que la phase OIDP (appelee "fluoresence variable") traduit la réduction de l'accepteur primaire d'électrons Q du PSII, qui produit un flux d'électrons à travers le 


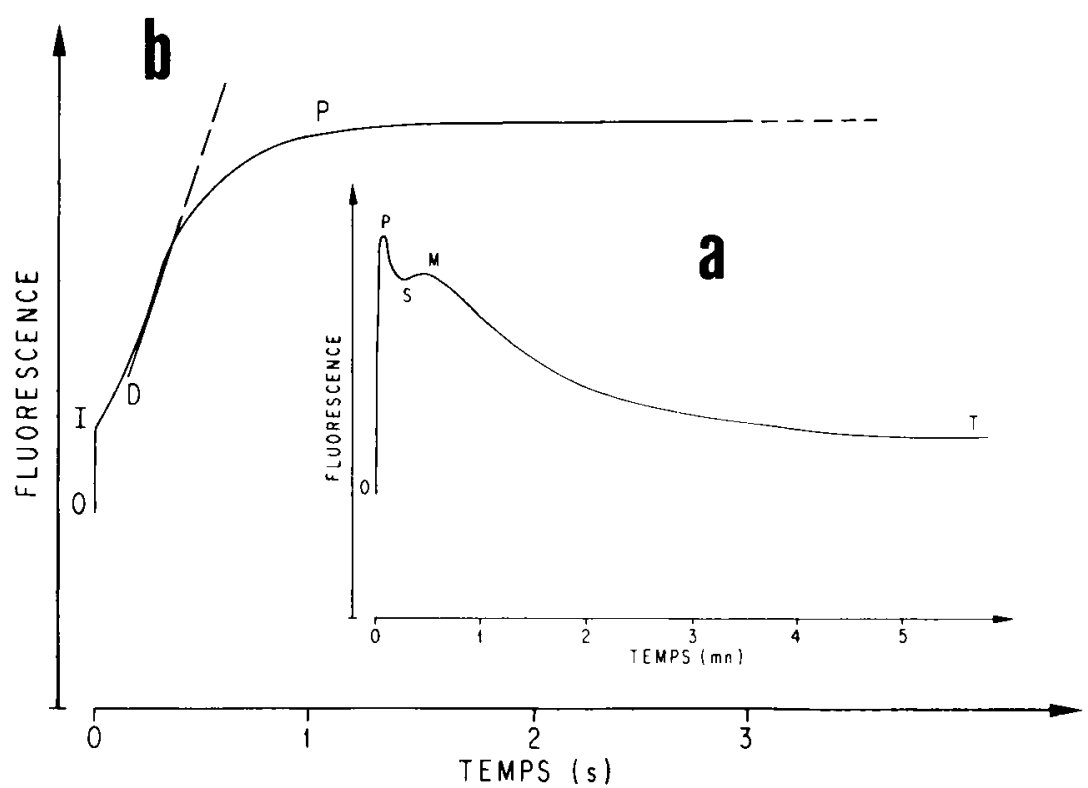

Fig 1. Cinétiques de l'induction de la fluorescence de la chlorophylle d'une feuille de $Q$ ilex non soumise au froid, réalisées avec : a) un enregistreur graphique potentiométrique, b) le dispositif d'acquisition et d'analyse de signaux rapides.

centre réactionnel P680 du PSII et provoque ainsi un accroissement de la fluoresence. La phase décroissante suivante ("quenching") est un phénomène complexe et a été attribuée à 3 facteurs : la re-oxydation de $Q$, la creation d'un niveau d'énergie élevé des thylakoïdes et la transition état l-état II. En particulier, dans une plante verte, un stress affectant le métabolisme photosynthetique affectera le rendement de la fluorescence variable. Smillie et Hetherington (1983) ont montré que ce rendement est d'autant plus faible que les accepteurs primaires du PSII sont dans l'état oxydé et capables d'extraire l'énergie du centre actif et se prête bien à l'étude de la tolérance au stress de plantes cultivées : plus pécisément, la vitesse maximale FR de l'accroissement de la fluorescence de la chlorophylle, mesurable par la pente après le point $D$, est à considérer (fig 1b). Sans toutefois analyser un impact direct sur le processus photosynthétique, ce parametre traduit bien, en effet, les dommages cellulaires, avant même l'observation dans la coloration de tout signe de nécrose : sa sensibilité au traitement par le froid ne semble pas avoir été retrouvée sur les autres caractères de la cinétique d'induction.

L'appareillage utilisé a été décrit par Méthy et Salager (1989). Ce dispositif de laboratoire est constitué essentiellement, pour l'excitation, d'une lampe aux halogenes et d'un filtre passe-bande sélectionnant la région spectrale $350-600 \mathrm{~nm}$; la détection est réalisee au travers d'un filtre passe-haut (longueur d'onde à $50 \%$ du maximum de transmission : $660 \mathrm{~nm}$ ) par un photomultiplicateur connecté à un micro-ordinateur équipé pour l'acquisition et l'analyse de signaux rapides. Les échantillons étudiés étaient, durant les mesures, disposes sur un buvard humide dans une chambre-support thermostatable.

Ces mesures ont été réalisées en 2 phases suffisamment brèves pour limiter l'effet d'une évolution de la teneur en chlorophylle. Pour l'étude des feuilles de 1 an, les échantillons ont été prélevés du 18 au 20 janvier 1988. Les feuilles de 1 mois ont été récoltées entre le 16 et le 18 mai 1988, sur les 2 mêmes sujets que précédemment ( 1 pour chaque espèce), poussant côte à côte. 
Les températures moyennes de l'air observees dans les 10 j précédant les mesures sont restées comprises entre $6,5{ }^{\circ} \mathrm{C}$ et $12,4^{\circ} \mathrm{C}$ (feuilles de 1 an) et $16,3^{\circ} \mathrm{C}$ et $19,6^{\circ} \mathrm{C}$ (feuilles de 1 mois). Les échantillons ont toujours été prélevés sur la même exposition. Les 2 individus, d'un âge estimé à plus de 30 ans, avaient été choisis parmi une population croissant spontanement sur le terrain d'expériences, situé à $100 \mathrm{~m}$ du centre Emberger, à Montpellier.

Les matins de chaque expérimentation, un rameau de $50 \mathrm{~cm}$ de longueur était prélevé pour chaque espèce, ceci afin de limiter l'effet de la superposition d'un stress hydrique, puis transporté au laboratoire.
Avant de déposer les rameaux, à l'obscurité, dans une enceinte cryogène, réglée dès la veille a la température désirée, des feuilles n'ayant subi aucun traitement au froid étaient prélevées, mises à l'obscurité pendant $30 \mathrm{~min}$ (duree d'adaptation trouvée suffisante pour éviter un effet éventuel sur la cinétique d'induction), puis immédiatement analysées par fluorescence. La réponse de leur systeme photosynthétique servira de référence parmi celles des systèmes soumis au froid.

Les essais au froid ont combiné à la fois temperatures et durées d'exposition (tableau I); les feuilles étant, lors de l'analyse de fluorescence, disposées la face abaxiale

Tableau I. Nombre de répétitions effectuées pour les différentes combinaisons de froid et de durées d'exposition pour chacune des espèces étudiées $(Q i=Q$. ilex; $Q c=Q$. coccifera), pour les feuilles âgées de 1 an (VF= vieilles feuilles) et pour les feuilles âgées de 1 mois seulement ( $\mathrm{JF}=$ jeunes feuilles). Température ambiante: $\mathrm{VF}=Q$. ilex: $6 ; Q$. coccifera: $4 ; \mathrm{JF}=$ Q. ilex: $3 ; Q$. coccifera: 3 .

\begin{tabular}{|c|c|c|c|c|c|c|c|c|}
\hline \multirow{3}{*}{$\begin{array}{c}\text { Durées } \\
\text { D'exposition }\end{array}$} & \multicolumn{8}{|c|}{ Temperatures (en ${ }^{\circ} \mathrm{C}$ ) } \\
\hline & \multicolumn{2}{|c|}{-5} & \multicolumn{2}{|c|}{-10} & \multicolumn{2}{|c|}{-15} & \multicolumn{2}{|c|}{-20} \\
\hline & Qi & $Q c$ & $Q i$ & $Q c$ & $Q i$ & $Q C$ & $Q i$ & $Q c$ \\
\hline \multicolumn{9}{|l|}{15 minutes } \\
\hline $\begin{array}{l}\text { VF } \\
\text { JF }\end{array}$ & $\begin{array}{l}3 \\
2\end{array}$ & $\begin{array}{l}5 \\
2\end{array}$ & $\begin{array}{l}2 \\
3\end{array}$ & $\begin{array}{l}2 \\
2\end{array}$ & 3 & 4 & 2 & 2 \\
\hline \multicolumn{9}{|l|}{30 minutes } \\
\hline VF & 5 & 6 & 3 & 3 & 4 & 3 & 3 & 2 \\
\hline JF & 3 & 1 & 1 & 1 & & & & \\
\hline \multicolumn{9}{|l|}{1 heure } \\
\hline VF & 4 & 3 & 4 & 4 & 3 & 3 & 4 & \\
\hline JF & 3 & 2 & & & & & & \\
\hline \multicolumn{9}{|l|}{2 heures } \\
\hline VF & 3 & 4 & 3 & 3 & 3 & 3 & 3 & \\
\hline \multicolumn{9}{|l|}{3 heures } \\
\hline VF & & & 2 & 2 & 3 & & & \\
\hline \multicolumn{9}{|l|}{5 heures } \\
\hline VF & 5 & 4 & & & 3 & & & \\
\hline \multicolumn{9}{|l|}{7 heures } \\
\hline VF & 5 & 5 & & & & & & \\
\hline
\end{tabular}


sur le support, thermostaté de manière à éviter toute remontée en température. Compte tenu des résultats en cours d'expérience et de l'absence de toute réaction à partir de certaines combinaisons, les expérimentations basées sur de longues durées à basse température n'ont pas été réalisées.

\section{RÉSULTATS}

\section{Feuilles âgées de 1 an}

Pour les 2 types de chêne, la vitesse de fluorescence variable définie cidessus, qui traduit l'activité du PSII, semble relativement peu affectée par plusieurs heures d'exposition à $-5^{\circ} \mathrm{C}$ comme à $-10{ }^{\circ} \mathrm{C}$ (fig $2 \mathrm{a}$ et b). En revanche, à $-15^{\circ} \mathrm{C}$ et surtout à $-20^{\circ} \mathrm{C}$, il y a une réduction très rapide de l'activité photosynthétique, $Q$ ilex apparaissant comme le plus résistant des 2 .

Pour quantifier la tolérance au froid, on peut considérer le temps correspondant à une certaine réduction de la vitesse de fluorescence variable (par exemple $90 \%$ ).

Il est à remarquer que, pour la température extrême de $-20^{\circ} \mathrm{C}$, ce temps est environ 2 fois plus élevé pour $Q$ ilex que pour $Q$ coccifera.

A $-5{ }^{\circ} \mathrm{C}$ comme à $-10^{\circ} \mathrm{C}$, les 2 espèces se comportent de manière semblable, l'impact du froid tout d'abord constaté semblant diminuer après $1 \mathrm{~h}$ d'exposition pour s'annuler à peu près complètement ensuite. Cette propriété pourrait traduire une sorte d'accoutumance de la feuille. En outre, les temps d'exposition relativement courts ne permettent pas de négliger un éventuel gradient thermique au sein de la feuille.

L'impact des basses températures est tel que les dommages subis par le système semblent généralement irréversibles. Après avoir vérifié que, à la température ambiante, une feuille détachée du rameau $9 \mathrm{~h}$ auparavant présentait la même cinétique qu'une feuille analysée immédiatement, des tests complémentaires ont été effectués sur 1 feuille des 2 espèces exposées préalablement durant $15 \mathrm{~min}$ à $-5^{\circ} \mathrm{C}$ et qui font l'objet de la figure 2. Après un nouveau séjour de $30 \mathrm{~min}$ à $-5^{\circ} \mathrm{C}$, les cinétiques observées sur ces feuilles sont comparables à celles obtenues sur des feuilles soumises seulement $30 \mathrm{~min}$ à $-5^{\circ} \mathrm{C}$.

Les dommages peuvent se poursuivre au-delà de la période d'exposition au froid et être irréversibles, comme il ressort de mesures effectuées sur des feuilles de $Q$ ilex exposées durant $3 \mathrm{~h}$ à $-15^{\circ} \mathrm{C}$, puis placées durant $2 \mathrm{~h}$ à la température ambiante (3 répétitions). Un repos de $5 \mathrm{~h}$ après une exposition de $2 \mathrm{~h}$ à $-20{ }^{\circ} \mathrm{C}$ a permis d'aboutir aux mêmes conclusions (2 répétitions) : le système chlorophyllien ne récupère donc pas ses capacités assimilatrices.

\section{Feuilles de 1 mois}

Les effets décrits ci-dessus sont amplifiés sur ces dernières. Les résultats rassemblés dans le tableau II montrent là encore une plus grande sensibilité de $Q$ coccifera, le système photosynthétique devenant totalement inopérant en moins de 30 min dès $-5^{\circ} \mathrm{C}$.

Comme précédemment, des tests d'irréversibilité ont été réalisés. Une feuille de $Q$ ilex et une de $Q$ coccifera ont été soumises à un traitement de $-5^{\circ} \mathrm{C}$ pendant $1 \mathrm{~h}$, puis laissées au repos à la température ambiante pendant 1 h et analysés à nouveau: aucune réponse n'a été observée. 

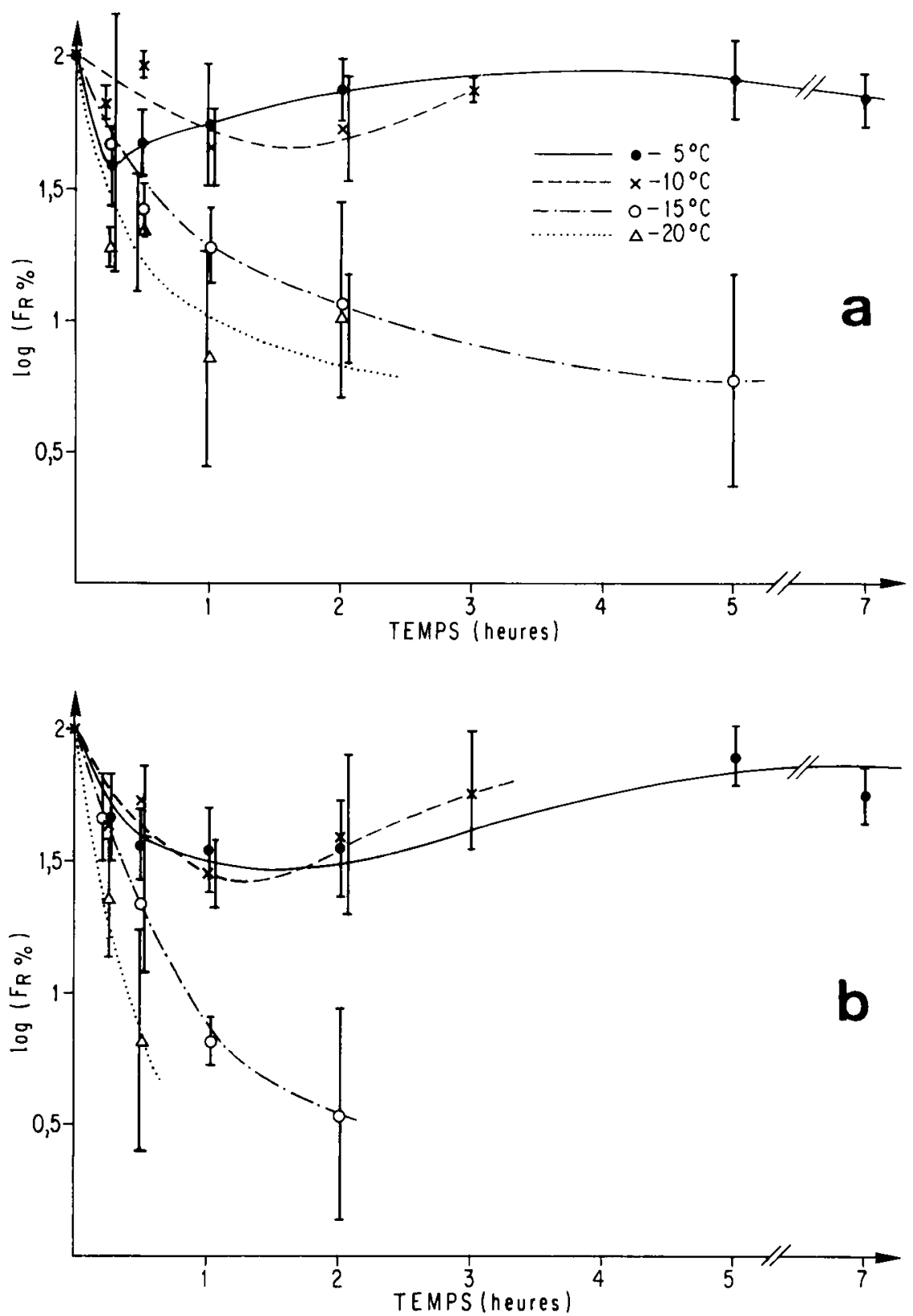

Fig $2 \mathbf{a}$ et $\mathbf{b}$. Effets du froid et de sa durée sur la vitesse de fluorescence variable ramenée à sa valeur de référence (FR) pour des feuilles de 1 an : a) de $Q$ ilex, b) de $Q$ coccifera. Les barres verticales correspondent à l'intervalle de confiance à $5 \%$. 
Tableau II. Effet du froid et de sa durée sur la fluorescence variable des feuilles de 1 mois de $Q$. llex et $Q$. coccifera.

\begin{tabular}{|c|c|c|c|}
\hline \multirow{2}{*}{$\begin{array}{c}\text { Températures } \\
\left({ }^{\circ} \mathrm{C}\right)\end{array}$} & \multirow{2}{*}{$\begin{array}{c}\text { Durées } \\
\text { d'exposition } \\
\text { (min) }\end{array}$} & \multicolumn{2}{|c|}{$\begin{array}{c}\log \left(F_{R} \%\right) \\
\text { (moyennes } \pm \text { erreurs standard })\end{array}$} \\
\hline & & $Q$ ilex & $Q$ coccifera \\
\hline Température ambiante & & 2 (référence) & 2 (référence) \\
\hline-5 & $\begin{array}{l}15 \\
30 \\
60\end{array}$ & $\begin{array}{c}1,94 \pm 0,26 \\
1,40 \pm 0,20 \\
0\end{array}$ & $\begin{array}{c}1,61 \pm 0,29 \\
1,06 \\
0\end{array}$ \\
\hline-10 & $\begin{array}{l}15 \\
30\end{array}$ & $\begin{array}{l}0 \\
0\end{array}$ & $\begin{array}{l}0 \\
0\end{array}$ \\
\hline
\end{tabular}

\section{DISCUSSION ET CONCLUSION}

Les résultats mis en évidence pour caractériser la résistance au froid des feuilles de $Q$ ilex et $Q$ coccifera sont en accord avec ceux obtenus, parmi d'autres sclérophylles méditerranéennes par Larcher (1981). L'épaisseur des feuilles analysées rend progressive l'action du gel : les résultats présentés, qui font intervenir la durée d'application de ces températures, précisent que chez les feuilles adultes le système chlorophyllien de $Q$ ilex et $Q$ coccifera peut supporter $-15^{\circ} \mathrm{C}$ pendant $5 \mathrm{~h}$ ou $2 \mathrm{~h}$, respectivement ; à $-20{ }^{\circ} \mathrm{C}$, ces durées sont réduites à 30 et $15 \mathrm{~min}$. L'effet est plus accentué sur les feuilles de 1 mois où une température de $-5{ }^{\circ} \mathrm{C}$, appliquée durant $1 \mathrm{~h}$, suffit à bloquer le processus.

Par ailleurs, l'application prolongée de basses températures entraîne des dégâts irréversibles dans le système chlorophyllien puisque des feuilles sou- mises à ces traitements puis laissées au repos à température ambiante ne récuperent pas leur aptitude à la photosynthèse.

Les mesures confirment, du point de vue de l'activité photosynthétique, la plus grande sensibilité au froid de $Q$ coccifera. Ces résultats ne doivent certes pas être superposés, sans précaution, à l'aire de répartition des 2 espèces, laquelle est en fait la résultante de diverses caractéristiques fonctionnelles. Cependant, les températures (inférieures, parfois, de $13^{\circ} \mathrm{C}$ ) donnees par Larcher (1981) pour la résistance au froid des autres organes végétatifs indiquent bien que c'est essentiellement la destruction de l'appareil chlorophyllien qui limite l'extension des espèces méditerranéennes. Si le système chlorophyllien est tué, l'espèce doit produire de nouvelles feuilles à partir des réserves accumulées dans les organes, ce qui la pénalise. Dans le Bassin méditerranéen, d'après Le Houerou 
(1974), l'extension de $Q$ coccifera serait limitée par l'isotherme $+1^{\circ} \mathrm{C}$ (température correspondant à la moyenne des températures minimales du mois le plus froid, avec $30 \mathrm{j}$ de gelée possible en hiver), tandis que l'isotherme $-2{ }^{\circ} \mathrm{C}$ limiterait l'aire de $Q$ ilex (60 j de gelée possible en hiver). En outre, la dispersion des données climatologiques disponibles ne permet pas toujours d'obtenir un diagnostic local précis.

Les résultats présentés ci-dessus, qui ne correspondent qu'à l'effet intrinsèque du froid, indépendamment de toute possibilité d'interaction avec la lumière (photo-inhibition) reflètent la sensibilité du système photosynthétique pour 2 stades de développement distincts. Ces observations doivent être considérées comme des indications pour mieux apprécier la capacité de survie des 2 espèces; cette dernière est en effet liée à l'impact du froid sur chacun des organes constitutifs et sur leur faculté de récupération (Larcher et Mair, 1969 ; Sakai et Larcher, 1987). L'intérêt de telles indications apparaît notamment lors de la gestion des taillis.

\section{REMERCIEMENTS}

Nous remercions plus particulièrement $\mathrm{JL}$ Salager pour la mise au point du logiciel d'acquisition et d'analyse de signaux rapides et $\mathrm{F}$ Jardon pour sa contribution à la réalisation du dispositif.

\section{RÉFÉRENCES}

Eckardt FE, Heim G, Méthy M, Sauvezon R (1975) Interception de l'énergie rayonnante, échanges gazeux et croissance dans une forêt méditerranéenne à feuil- lage persistant (Quercetum ilicis). Photosynthetica (Prague) 9, 145-156

Kautsky H, Hirsch A (1931) Neue Versuche zur Kohlenstoffassimilation. Naturwissenschaften 19,964

Larcher W (1970) Kälteresistenz und Überwinterungsvermögen mediterraner $\mathrm{Holz}$ pflanzen. Oecol Plant 5, 267-286

Larcher W (1981) Low temperature effects on mediterranean sclerophylls : an unconventional viewpoint. In : Components of Productivity of Mediterranean-Climate Regions : Basic and Applied Aspects (Margaris NS and Mooney HA, eds). W Junk, La Haye, 259-266

Larcher W, Mair B (1969) Die Temperaturresistenz als ökophysiologisches Konstitutionsmerkmal. I. Quercus ilex und andere Eichnarten des Mittelmeergebietes. Oecol Plant 4, 347-376

Lavorel J, Etienne AL (1977) in vivo chlorophyll fluorescence. In : Topics in Photosynthesis, 2 (Barber J ed). Elsevier, Amsterdam, 203-268

Le Houerou HN (1974) Fire and vegetation in the Mediterranean basin. Proc Tall Timbers Fire Ecol Conf 13, 237-277

Lichtenthaler HK, Rinderle U (1988) The role of chlorophyll fluorescence in the detection of stress conditions in plants. CRC Crit Rev Anal Chem 19, Suppl 1, 529-585

Methy M., Salager JL (1989) A microcomputer based fast data acquisition system for in vivo measurements of stress effects in crop plants by chlorophyll fluorescence induction. Comput Electron Agric, 121128

Sakai A, Larcher W (1987) Frost survival of plants, responses and adaptation to freezing stress. Ecol Stud 62, 321pp

Smillie RM, Hetherington SE (1983) Stress tolerance and stress-induced injury in crop plants measured by chlorophyll fluorescence in vivo. Plant Physiol 72, $1043-1050$

Trabaud L (1981) Man and fire: impacts on Mediterranean vegetation. In : Ecosystems of the World. 11. Mediterraneantype Shrublands (di Castri F, Goodall DW, Specht RL, eds). Elsevier, Amsterdam, 523-537 